\title{
New Statistical Methods for Analysis of Large Surveys: Distributions and Correlations
}

\author{
Vahé Petrosian \\ Center for Space Science and Astrophysics, Varian 302c, Stanford \\ University, Stanford, CA 94305-4060
}

\begin{abstract}
The aim of this paper is to describe new statistical methods for determination of the correlations among and distributions of physical parameters from a multivariate data with general and arbitrary truncations and selection biases. These methods, developed in collaboration with B. Efron of Department of Statistics at Stanford, can be used for analysis of combined data from many surveys with different and varied observational selection criteria. For clarity I will use the luminosity function of AGNs and its evolution to demonstrate the methods. I will first describe the general features of data truncation and present a brief review of past methods of analysis. Then I will describe the new methods and results from simulations testing their accuracy. Finally I will present the results from application of the methods to a sample of quasars.
\end{abstract}

\section{INTRODUCTION}

One of the important ways of testing the models of AGN, or any other astrophysical source, is through the investigations of the distributions, ranges, and more importantly the correlations among, the relevant physical characteristics, such as luminosity, spectrum, redshifts or distances. A reliable determination of these features requires large samples. As evident from papers presented in this proceedings the samples are becoming larger and larger. Combining the samples, however, is a very challenging task, because different samples are obtained by different instruments and techniques, so that they suffer from different and varied selection biases and data truncations. Overcoming these biases requires care.

The primary goal of this paper is to describe some of the relatively new methods we have developed at Stanford over the past decade (Efron \& Petrosian 1992, 1999). These methods are very general and are applicable to any data with well defined but arbitrary truncations. We have applied these to various astrophysical data such as solar flares (Lee, Petrosian, \& McTiernan 1993, 1995), gamma-ray burst (see e.g. Lloyd, Petrosian, \& Mallozzi 2000) and quasars (Meloney \& Petrosian 1999). Instead of using abstract mathematical symbols, the method will be demonstrated using the luminosity function of AGNs and its cosmological evolution, i.e. its variation with redshift $z ; \Psi(L, z)$. Without loss of generality, we can write the luminosity function as

$$
\Psi(L, z)=\rho(z) \psi\left(L / g(z), \alpha_{i}\right) / g(z)
$$


where $\rho(z)$ describes the co-moving density evolution and $g(z)$ (with $g(0)=1$ ) describes the luminosity evolution of the population with $L_{o}=L / g(z)$ as the luminosity adjusted to its present epoch value; $\psi\left(L_{o}, \alpha_{i}\right)$ gives the local luminosity function. Here I explicitly include the shape parameters $\alpha_{i}$, which could also vary with redshift. A surprising result has been the absence of evidence for a strong shape evolution. In this paper I ignore such effects and concentrate on the determination of the the density and luminosity evolution functions $\rho(z)$ and $g(z)$.

In the next section we describe various kind of truncations and in $\S 3$ give a brief summary of some of the past methods used for this kind of analysis. The new methods and their accuracy are described in $\S 4$ and a sample of results from application to AGNs are summarized in $§ 5$.

\section{Types of Truncations}

The left panel of Figure 1 shows a set of arbitrary data points labeled as luminosity $L$ and redshift $z$ and several generic truncations. The distribution may be truncated parallel to the axis as shown by the dotted lines. This only limits the observed ranges of the variables but does not introduce any bias. This kind of data will be referred to as untruncated data. However, this is rarely the case for astronomical data, and in general, one is dealing with cases where the truncation is not parallel to the axis. The simplest and most common case is when the data suffers a one-sided truncation from below as shown by the solid curve. This is the case for magnitude or flux limited samples; $L \geq 4 \pi d_{L}^{2}\left(\Omega_{i}, z\right) f_{\min }$, where $f_{\min }$ is the limiting flux and $d_{L}\left(\Omega_{i}, z\right)$ is the luminosity distance at $z$ for an assumed cosmological model represented by $\Omega_{i}$. In some cases the data may be truncated from above as shown by the dashed curve. The statistical methods do not distinguish between truncation from above or below. However, the data analysis is affected when there are truncations both from above and below. This is the case for some AGN samples. The situation becomes even more complex when the truncation boundaries are not monotonic, or when one tries to combine samples with different truncations, say different upper and lower flux limits. Such variation may be present even within a given catalog where data taken at different times and directions in the sky may have different limits. The most general truncation is when each data point, say $\left[L_{i}, z_{i}\right]$, has its individual upper and lower limits, $L_{i}^{-}<L_{i}<L_{i}^{+}$and $z_{i}^{-}<z_{i}<z_{i}^{+}$, as shown by the large cross for one point on Figure 1. The methods we have developed can treat this most general truncation.

\section{A Brief Historical Review}

The process of determination of the distribution of physical characteristics from truncated data has a long history starting with first and rudimentary observations of stars in the disk of our galaxy. Here I will touch upon some of the relevant highlights. A more detailed discussion can be found in the references cited below and in a review article (Petrosian 1992). I will limit the discussion to works aimed at determination of the luminosity function and spatial distribution of sources; $\Psi(L, r)$ from a magnitude limited data (one sided truncation) 

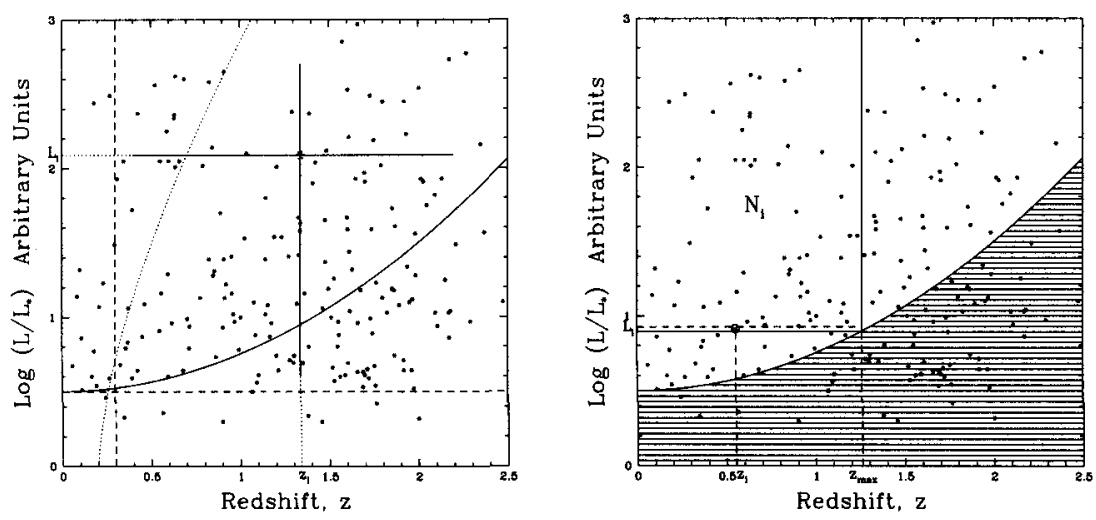

Figure 1. Left Panel: Demonstration of various types of data truncations: Parallel to axis (dotted lines), from below (the solid curve), from above (the dashed curve), and a general truncation when each data point has its specific observable range (shown by the cross for only one of the points). Right Panel: Description of the constructs used for evaluation of the univariate distribution from uncorrelated data truncated from below. The large box contains $N_{i}$ sources in the comparable or associated set of the source $i$ located in the thin rectangle defined by the dashed horizontal line.

as shown by the right panel in Figure 1. Almost all the methods I will describe ignore possible correlations between the variables and assume that these are independent. In the case of the luminosity function this means that $g(z)=1$, so that the bivariate distribution is separable; $\Psi(L, r)=\psi(L) \rho(r)$. Unfortunately this often unjustified simplification is prevalent even today. This unnecessary assumption often can lead to erroneous conclusions. As stressed below the first task must be testing the bivariate distribution for correlations between the variables.

In general, most of the methods can be divided into two categories, parametric or non parametric. In the former one assumes a parametric form for the two functions $\psi(L)$ and $\rho(r)$. In the non parametric methods one often ends up with a description for the cumulative functions

$$
\phi(L)=\int_{L}^{\infty} \psi\left(L^{\prime}\right) d L^{\prime}, \sigma(r)=\int_{0}^{r}(d V / d r) \rho\left(r^{\prime}\right) d r^{\prime},
$$

where $V(r)$ is the volume of space (included in the observation) from the origin to distance $r$. In these relation $r$ could be any measure of distance including redshift, look back time etc.

\subsection{Parametric Methods}

The first methods were developed for investigation of the luminosity function and spatial distribution of stars perpendicular to the galactic disk. These and subsequent applications to other sources have come to be known as correction 
for Malmquist Bias after Malmquist (1922); Eddington (1915, 1940) also describes this method. A more recent description can be found in Trumpler \& Weaver (1953). These early works dealing with the distribution of stars assume a Gaussian distribution of absolute magnitudes (i.e. a log-normal luminosity function) and a Gaussian spatial distribution perpendicular to the disk. Because of the truncation there is absence of low luminosity stars at large distance so that the raw observed distributions of $L$ and $r$ are biased. The essence of the method was to correct for this bias. It turns out that the method used was sound but the final results were incorrect because of the erroneous assumptions about the forms of the distributions. We know today that the luminosity function of stars is best represented by a broken power law and that the fall of the stars perpendicular to the disk is exponential and not Gaussian. This demonstrates the shortcoming of this and other parametric methods. Similar parametric methods have been used for extragalactic sources (galaxies and quasars) first by Neymann \& Scott (1959) and in numerous works ever since.

\subsection{Non-parametric Methods}

One of the most commonly non parametric methods used is the so-called $V / V_{\max }$ method first described by Kafka (1966) soon after the discovery of the quasars but used most successfully by Schmidt (1968). Independence is again assumed and the presence or absence of density evolution is tested by a single moment, namely the average value of $V / V_{\max }$, where $V_{\max }$ is the volume up to the maximum redshift (or distance) that a source of luminosity $L$ can be visible given the limiting flux $f_{\min } ; L=4 \pi d_{L}^{2}\left(\Omega_{i}, z_{\max }\right.$ ) (see Figure 1 ). In the absence of evolution one expects a value of 0.5 for this average. A more general method was described later by Avni \& Bahcall (1980). Of course, one need not be limited only to one moment of the distribution. More information can be obtained by examining the distribution of $\left(V / V_{\max }\right)_{i}$ of the whole data set.

Schmidt (1968) also described a method for determination of $\phi(L)$, which was later dubbed as the "Schmidt Estimator" by Felten (1976). It is straightforward to show that in absence of evolution, i.e. for a uniform spatial distribution, the contribution to this cumulative luminosity function of each source is proportional to $V_{\max , i}^{-1}$. It is also easy to show that if there is evolution this contribution is proportional to $\sigma_{\max , i}^{-1}$ defined in equation $(2) ; \sigma$ was denoted as $V^{\prime}$ by Schmidt.

Most other methods employ binning, which simplifies the problem conceptually but has several shortcomings, the primary being loss of data points in the incomplete bins at the truncation boundaries. Examples of these are anlysis by Nicole \& Segal (1978, 1983), Turner (1979) and Choloniewski (1986, 1987). As shown by Petrosian (1986), it turns out that all these procedures, in the limit of one source per bin reduce to Lynden-Bell's (1979) $C^{-}$method. For a detailed comparison of these methods see Petrosian (1992).

\subsection{The General Method}

The right panel of Figure 1 depicts two boxes. Let us assume that number of data points in the narrow box is $n(L) d L$ and the number in the big box, excluding the narrow region is $N(L)$. It is easy to show that if the variables are independent, i.e. the luminosity function is separable in $L$ and $z$, then 


$$
\frac{n(L) d L}{N(L)}=\frac{\sigma\left(z_{\max }\right) \psi(L) d L}{\sigma\left(z_{\max }\right) \phi(L)}=-d \ln \phi(L)
$$

In the limiting case of one object per (narrow) bin, $n(L)=\delta\left(L-L_{i}\right)$ and $N(L)=N_{i}+\Theta\left(L-L_{i}\right)$, where $\Theta$ is the Heviside step function.

Integration of the above equation then shows that the cumulative luminosity function $\phi(L)$ increases by $\delta \ln \phi(L)=\ln \left(1+N_{i}^{-1}\right)$ going across the source at $L_{i}$. Thus, the cumulative luminosity function can be build in increments starting, say from the highest observed luminosity $L_{1}$, as

$$
\phi(L)=\phi\left(L_{1}\right) \prod_{L_{i}>L} \ln \left(1+N_{i}^{-1}\right) .
$$

The set of $N_{i}$ sources in the big box are referred to as the comparable or the associated set of the source with luminosity $L_{i}$. It is clear that because of the complete mathematical symmetry between the two variables we can get an identical expression for the cumulative distribution $\sigma(z)$. The (different) associated sets are defined in a similar manner.

In the next section I will describe the extension of this method to more complex truncations. I will consider only bivariate distributions. The generalizations to multivariate distributions is straightforward.

\section{The New Methods}

The complete description of the distributions is a two step process. The first step is to determine whether the two variables are correlated or they are independent, and if correlated, then find a way to account for this correlation. The latter step can be done only parametrically. For the luminosity function it entails the determination of the form of the luminosity evolution function $g(z)$ such that a redefined luminosity $L_{0}=L / g(z)$ is uncorrelated with, or is independent of, $z$. The second step is to determine the univariate distributions of the independent variables $z$ and $L_{0}$.

\subsection{Untruncated Data}

If $z$ and $L$ are independent then the rank $R_{i}$ of $z_{i}$ (or $L_{i}$ ) in an untruncated sample (i.e. a sample truncated parallel to the axes; $z>z_{\min }$ and $L>L_{\min }$ ) will be distributed uniformly between 1 and $N$ with an expected mean $E=\frac{1}{2}(N+1)$ and variance $V=\frac{1}{12}\left(N^{2}-1\right)$. We may then normalize $R_{i}$ to have a mean of 0 and a variance of 1 by defining the statistic $T_{i}=\left(R_{i}-E\right) / V$. The hypothesis of independence is then rejected or accepted using a statistics based on the distribution of the $T_{i}$. The quantity

$$
\tau=\frac{\sum_{i}\left(R_{i}-E\right)}{\sqrt{\sum_{i} V}}
$$

is one choice of such a test statistic with a mean of 0 and a variance of 1 . The hypothesis of independence is rejected if $\left|\tau_{\text {data }}\right|$ is too large (e.g. $\left|\tau_{\text {data }}\right| \geq 1$ for rejection of independence at the $1 \sigma$ level). This $\tau$ is equivalent to Kendell's $\tau$ statistic (see, e.g. Press et al. 1990) 
If it turns out that $\left|\tau_{\text {data }}\right| \leq 1$ and that the variables are independent, then the determination of the univariate distributions in each variable is obtained ignoring the value of the other. For example, the cumulative luminosity function will be described by the histogram $\phi\left(L_{i+1}>L>L_{i}\right)=i$. However, if the variables are correlated, one must then carry out a transformation to remove the correlation by some parametric function, say $L_{0}=L / g(z)$. It is important to note that the transformed data will now appear truncated in the $L_{0}-z$ plane with $L_{0, \min }=L_{\min } / g(z)$, which is no longer parallel to the $z$ axis. The use of the method described by equation (3) is then required to obtain the univariate distributions.

\subsection{Data with One-sided Truncation}

A straightforward application of the above method to a truncated data will clearly give a false correlation signal. Efron \& Petrosian (1992), and independently Tsai (1990), describe how this method can be applied to data with onesided truncation. The above procedure is modified as follows. For each object define a new comparable or associated set

$$
J_{i}=\left\{j: L_{j}>L_{i}, L_{j}^{-}<L_{i}\right\},
$$

where $L_{j}^{-}=4 \pi d_{L}^{2}\left(\Omega_{i}, z_{j}\right) f_{\min }$. It is easy to see that this is the same set defined above (Figure 1) as the big box containing $N_{i}$ sources. (Note that the set defined in Efron \& Petrosian (1992) includes the object $i$ in question.) This is the largest subset of luminosity and volume limited data that can be constructed for each point $\left(L_{i}, z_{i}\right)$. If $z$ and $L$ are independent then we expect the rank $R_{i}$ of $z_{i}$ (or $L_{i}$ ) in this limited set, not in the whole sample to be uniformly distributed between 1 and $N_{i}$. The rest of the procedure follows the steps described above.

Similarly the determination of the univariate distributions will require the use of the method described in the previous section for the transformed and uncorrelated variables. Note again that the truncation boundary also gets transformed in case there is a correlation between $L$ and $z$.

\subsection{Complex Truncations}

A generalization of the above method to doubly (or multiply) truncated data was developed by Efron \& Petrosian (1999), which is valid for the most general truncation $L_{i}^{-}<L_{i}<L_{i}^{+}$and $z_{i}^{-}<z_{i}<z_{i}^{+}$. The method is equivalent to the previous method, with the associated set defined as

$$
J_{i}=\left\{j: L_{j}>L_{i}, L_{i} \in\left(L_{j}^{-}, L_{j}^{+}\right)\right\} .
$$

In this case, however, the distribution of the rankings (or of $\tau$ ) is unknown. If the data are uncorrelated then $\tau$ must still have a mean of zero. But a bootstrap method using simulations based on the $\psi(L)$ obtained from the data, as described below, is required for the purpose of the estimation of the variance.

For doubly (or more complexly) truncated data the comparable set is not completely observed, thus a simple analytic method such as the one described in equation (4) is not possible. However, it turns out that a simple iterative procedure can lead to a maximum likelihood estimate of the distributions. Efron 
and Petrosian (1999) give a thorough description of this method; for a more brief and transparent description see Maloney \& Petrosian (1999).

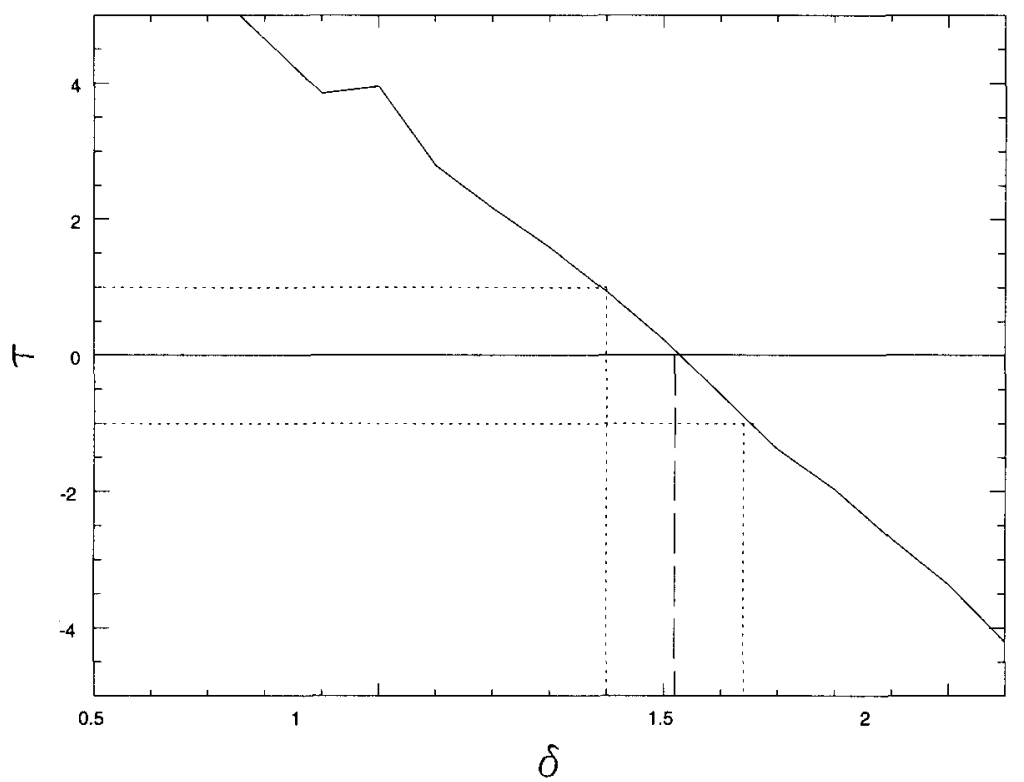

Figure 2. Variation of the $\tau$ statistics with exponent $\delta$ of the assumed correlation $\bar{y} \propto x^{-\delta}$. For the parent sample $\delta=1.5$.

\subsection{Tests of the Correlation Algorithm}

Lloyd et al. (2000) describe two simulations which test how well the above procedures reproduce known distributions. In one simulation the method was applied to a single sided truncation of an uncorrelated bivariate parent distribution. The rank test applied to the untruncated simulated points gave a value of $\tau=0.9$ But when applied to the truncated data without considering the effects of the truncation resulted in $\tau>5.0$, indicating the presence of a strong (of course false) correlation that is introduced by the truncation. However, when the test was carried out correctly by accounting for the effects of the truncation (as described above) it was found that $\tau=0.6$, recovering the fact that the variables were independent. In a second set the simulated parent sample had a strong anti correlation, with variables $y$ and $x$ correlated with average value of $\bar{y} \propto x^{-1.5}$. Application of the correlation to the untruncated randomly selected sample, as expected, gave a negative value for the statistic; $\tau<-5$. The data was truncated and the method applied blindly. This resulted in the value of $\tau=+2.5$, or a (false) positive correlation. However, when applied properly it gave a value of $\tau=-4.0$ indicating the presence of the anti correlation at 4 sigma level. Assuming a correlation of $\bar{y} \propto x^{-\delta}$, the data was transformed accordingly and the method applied to the new data. Figure 2 shows the variation of $\tau$ with 
the exponent $\delta$, from which we deduce a mean value and one sigma range of $\delta=1.51 \pm 0.11$. This is a strong support for the accuracy of the method.

Further simulations are required to test the methods for determination of the distribution and correlations for more complex truncations.

\section{Some Results from AGN Data}

Our application of these methods to a combine set of several optically selected samples of quasars are described in Maloney \& Petrosian (1999). Here is a brief summary.

- We found a strong correlation between luminosity and redshift, indicating the presence of a rapid luminosity evolution.

- The parametric model of luminosity evolution $(1+z)^{k^{\prime}}$ provides a better description of the data than the model $e^{k t(z)}$, where $t(z)$ is the look back time. Neither parameterization perfectly removes the correlation in all areas of the $L-z$ plane. In order to better model this evolution future analyses of quasar evolution could consider parametric forms, with more than one free parameter. Some of the more complex forms of evolution suggested in the literature, e.g. the used by La Franca \& Cristiani (1996) are equivalent with a luminosity evolution form that contains two independent free parameters.

Given the form of the luminosity evolution we make the simple transformation of all luminosities to their hypothetical present epoch values, $L_{0}=L / g(z)$, so that $L_{0}$ and $z$ are uncorrelated. This allows us to use our methods to determine the univariate distributions of $z$ (the density evolution) and $L_{0}$ (the local luminosity function).

- We find that the co-moving density of quasars also evolves, but its extent depends on the cosmological model. For example, for the Einstein-de Sitter model $\rho \sim(1+z)^{2.5}$ for low redshifts and rapidly declines as $\rho \sim(1+z)^{-5}$ for $z>$ 2. This is much slower evolution than is obtained when one (incorrectly) assumes a pure density evolution model; $g(z)=1$ (Schmidt 1968; Miyaji, Hasionger \& Schmidt 1998).

- The cumulative local luminosity function $\phi\left(L_{0}\right)$ has the double power law form found previously (Caditz \& Petrosian 1990, La Franca \& Cristiani 1996), with a break luminosity of $L_{*}=6 \times 10^{29} \mathrm{erg} /(\mathrm{sec} \mathrm{Hz})$, in the Einstein-de Sitter model. The power-law indices at the low and high luminosity ends are -1.5 and -2.3 in rough agreement with previous estimates. There is however, some evidence for evolution of the shape of the luminosity function. More data is required for a quantitative description of this evolution.

\section{References}

Avni, Y., \& Bahcall, J. N. 1980, ApJ, 235, 694

Caditz, D., \& Petrosian, V. 1990, Ap.J., 357, 326

Choloniewski, J. 1986, M.N.R.A.S., 192, 61

Choloniewski, J. 1987, M.N.R.A.S., 226, 273

Eddington, A. 1915, M.N.R.A.S., 73, 359 
Eddington, A. 1940, M.N.R.A.S., 100, 354

Efron, B., \& Petrosian, V. 1992, Ap.J., 399, 345

Efron, B., \& Petrosian, V. 1999, JASA, 94, 447

Felten, J.E. 1976, Ap.J., 207, 700

Kafka, P. 1967, Nature, 213, 346

La Franca, F., \& Cristiani, S. 1996, invited talk in Wide Field Spectroscopy (20-24 May 1996, Athens), Eds. M. Kontizas et al. astro-ph/9610017

Lee, T. T., Petrosian, V., \& McTiernan, J. M. 1993, 412, 401

Lee, T. T., Petrosian, V., \& McTiernan, J. M. 1995, 448, 915

Lloyd, M. N., Petrosian, V., \& Mallozzi, R. S. 2000, 534, 227

Lynden-Bell, D. 1971, M.N.R.A.S., 155, 95

Malmquist, K. G. 1920, Medd. Lund. Obs., Ser. 2, No. 22

Maloney, A. \& Petrosian, V. 1999, Ap.J., 518, 32

Miyaji, T. Husinger, G. \& Schmidt, M. 1998, A\&A, 369, 49

Neyman, J. \& Scott, E. L. 1959, Handbuch Der Physik, Vol 53, ed. S. Flugge, Springer-Verlag, Berlin, p. 416

Nicole, J. F. \& Segal, I. E. 1978, Ann. Phys. 113, 1

Nicole, J. F. \& Segal, I. E. 1983, Astr. Ap., 118, 180

Petrosian, V. 1986, Structure and Evolution of Active Galactic Nuclei, eds. Giuricin et al., D. Reidel Publ. Co., pp. 353-381

Petrosian, V. 1992, in Statistical Challenges in Modern Astronomy, Eds. E. D. Feigelson \& G. J. Babu, (New York: Springer-Verlag), p. 173

Press, W. H., Teukolsky, S. A., Vetterling W. T., \& Flannery, B. P., 1992. Numerical Recipes in FORTRAN, (Cambridge: Cambridge University Press)

Schmidt, M. 1968, Ap.J., 151, 393

Trumpler, R. J. \& Weaver, H. F. 1953 Statistical Astronomy, Dover, N.Y.

Tsai, W. 1990, Biometrika, 77, 169. 